\title{
On the feasibility of partial slip reversal and de-twinning during the cyclic loading of TWIP steel
}

Ahmed A. Saleh ${ }^{1 *}$, Bjørn Clausen², Donald W. Brown², Elena V. Pereloma ${ }^{1,3}$, Christopher H.J. Davies ${ }^{4}$, Carlos N. Tomé2, Azdiar A. Gazder ${ }^{3}$

${ }^{1}$ School of Mechanical, Materials and Mechatronic Engineering, University of Wollongong, NSW 2522, Australia

${ }^{2}$ Los Alamos National Laboratory, NM 87545, United States

${ }^{3}$ Electron Microscopy Centre, University of Wollongong, NSW 2500, Australia

${ }^{4}$ Department of Mechanical and Aerospace Engineering, Monash University, VIC 3800, Australia

\begin{abstract}
A recently modified Elasto-Plastic Self-Consistent (EPSC) model which empirically accounts for both intergranular and intragranular back stresses has been successfully used to simulate the cyclic (tension-compression) loading behaviour of an Fe-24Mn-3Al-2Si-1Ni-0.06C TWinning Induced Plasticity (TWIP) steel between strain limits of $\pm 1 \%$. Lattice strain measurements acquired via in-situ neutron diffraction were used to further validate the modelling results. An improved prediction of the pronounced Bauschinger effect during unloading is achieved when the reversibility of partial slip in the $\langle 112\rangle$ direction is accounted for. This result indicates a potential contribution of the stressinduced separation of partial dislocations to the observed early yielding at the low strain levels employed in this study. It also raises the possibility that de-twinning events could be operative during load reversal.
\end{abstract}

Keywords: TWIP, Neutron diffraction, Simulation and modelling, EPSC, Bauschinger effect.

* Corresponding author:

Ahmed A. Saleh

Phone: +612 42213034

Fax: +61242213662

E-mail: asaleh@uow.edu.au 


\section{Introduction}

TWinning Induced Plasticity (TWIP) steels comprise a stable face-centred cubic (fcc) austenite phase with low stacking fault energy $\left(\mathrm{SFE}=18-40 \mathrm{~mJ} / \mathrm{m}^{2}\right.$ ) that promotes deformation accommodation via twinning along with dislocation glide. This low SFE also facilitates the dissociation of perfect dislocations gliding on the $\{111\}$ plane in the $\langle 110\rangle$ direction into $\langle 112\rangle$ Shockley partials ( $a / 2[10 \overline{1}] \rightarrow a / 6[11 \overline{2}]+a / 6[2 \overline{1} \overline{1}])$ bounding stacking faults.

While the modelling of the monotonic deformation behaviour of TWIP steel has been the focus of numerous studies (see [1,2] and the references therein), the modelling of its cyclic (reverse) loading remains very limited. In this regard, we recently applied a combination of in-situ neutron diffraction and a modified Elasto-Plastic Self-Consistent (EPSC) modelling scheme to a TWIP steel subjected to cyclic (tension-compression) loading [3]. A pronounced Bauschinger effect was observed upon load reversal (or early yielding during unloading) and was attributed to a combination of intergranular residual stresses and intragranular sources of back stress such as dislocation pile-ups at the intersection of stacking faults. The modified EPSC model has been successfully used to simulate the macroscopic stress-strain response and the evolution of the lattice strains.

While our simulations in [3] considered both perfect slip and twinning (via the "twinning scheme" of Clausen et al. [4]), the potential contribution of partial slip to the overall mechanical response was not taken into account. The present study is therefore the first to assess the influence of deformation faulting or slip by partials during the cyclic loading of a TWIP steel, such that we provide a more holistic analysis of the contribution of perfect/partial slip and twinning to the overall deformation behaviour. The implications of slip reversal along the $\langle 112\rangle$ direction before and after the initiation of twinning are also discussed.

\section{Material and methods}

A cast slab of a $24 \mathrm{Mn}-3 \mathrm{Al}-2 \mathrm{Si}-1 \mathrm{Ni}-0.06 \mathrm{C}$ wt. $\%$ TWIP steel was $52 \%$ hot rolled, $42 \%$ cold rolled and then annealed at $850{ }^{\circ} \mathrm{C}$ for $300 \mathrm{~s}$ to obtain a fully recrystallized microstructure. A round tension/compression sample of $7.62 \mathrm{~mm}$ gage length and $2.54 \mathrm{~mm}$ diameter was machined with its gage length parallel to the rolling direction. In-situ neutron diffraction measurements during cyclic tension-compression loading between strain limits of $\pm 1 \%$ were performed on the SMARTS diffractometer at Los Alamos Neutron Science Center. Five complete tension-compression cycles were performed followed by a sixth tension half cycle. The changes in the individual peak positions were used to calculate the $\{\mathrm{hkl}\}$ specific residual lattice strains by $\varepsilon_{h k l}=\left(d_{h k l}-d_{h k l}^{0}\right) / d_{h k l}^{0}$, where, $d_{h k l}$ and $d_{h k l}^{0}$ are the instantaneous and unstrained lattice spacing, respectively. Further experimental and analytical details are given in [3]. 


\section{Elasto-plastic self-consistent modeling}

In the EPSC model [5], the elastic response of the individual grains is described by the single crystal elastic constants $\left(\mathrm{C}_{11}=161.5 \mathrm{GPa}, \mathrm{C}_{12}=110.3 \mathrm{GPa}\right.$ and $\left.\mathrm{C}_{44}=133.3 \mathrm{GPa}\right)$ [3], whereas the plastic response is described by activating the various deformation systems $(s)$ at predetermined values of the critical resolved shear stress (CRSS). The CRSS evolves with the total accumulated shear strain ( $\Gamma=\sum_{s} \Delta \gamma^{s}$ ) following an extended Voce hardening rule:

$$
\tau_{\text {for }}^{s}=\tau_{0}^{s}+\left(\tau_{1, f o r}^{s}+\theta_{1, f o r}^{s} \Gamma\right)\left[1-\exp \left(-\frac{\theta_{0, \text { for }}^{s} \Gamma}{\tau_{1, f o r}^{s}}\right)\right]
$$

Here $\tau_{\text {for }}^{s}$ represents the isotropic hardening associated with the non-directional accumulation of obstacles such as forest dislocations and/or deformation twin boundaries. Since the EPSC model allows for variations in stress and strain between different grains (orientations), it can inherently capture the effect of the intergranular stress but not the intragranular stress. Consequently, the model was recently modified ${ }^{1}$ to include a back stress term $\left(\tau_{b s}^{s}\right)$ in order to account for the kinematic hardening in individual slip systems as it reduces the resolved applied stress by the directional back stress arising from the pile-up of dislocations at various barriers [6]:

$$
\tau_{b s}^{s}=\left[\left(\tau_{1, b s}^{s}+x_{0}^{s}\right)+\theta_{1, b s}^{s}\left(\gamma^{s}-\gamma_{0}^{s}\right)\right]\left[1-\exp \left(-\frac{\theta_{0, b s}^{s}\left(\gamma^{s}-\gamma_{0}^{s}\right)}{\left(\tau_{1, b s}^{s}+x_{0}^{s}\right)}\right)\right]
$$

In Eqs. (1) and (2), $\tau_{0}^{s}$ and $\tau_{1}^{s}$ are the initial and back extrapolated CRSS and $\theta_{0}^{s}$ and $\theta_{1}^{s}$ are the initial and final asymptotic hardening rates for the forest hardening (for in Eq. (1)) and back stress (bs in Eq. (2)) formulations. The $\gamma_{0}^{s}$ and $x_{0}^{s}$ terms are the strain and stress "memory" parameters which are both initially set to zero during the first forward half cycle. Please refer to [3-6] for further details on the EPSC model.

The initial texture input for all simulations comprised 5000 discrete orientations calculated from the fully annealed texture. Two modelling cases ${ }^{2}$ have been applied as follows:

(i) Case I utilizes the twinning scheme of Clausen et al. [4] which was applied in [3], and included here for comparison with Case II. This scheme accounts for the stress relaxation associated with twin formation via the so called "finite initial fraction" approach; wherein the twin is assumed to grow to a fixed volume fraction of its parent grain at the nucleation stage. Here the plastic shear of the twinning

\footnotetext{
1 Please note that the modified EPSC model strictly deals with load reversal at small strains; where the Bauschinger effect is mostly related to the reversal of dislocation motion.

2 In both cases, equal latent hardening is imposed such that all deformation systems are assumed to contribute equally to the hardening of each other.
} 
system generates a back stress between the parent and the twin due to the constraint of the surrounding polycrystalline aggregate.

In Case I, $24\{111\}\langle 110\rangle$ perfect slip systems (counting both forward and reverse slip directions) and $12\{111\}\langle 112\rangle$ forward twinning systems (due to the unidirectional nature of twinning) were introduced into the EPSC model.

(ii) Case II assesses the contribution of deformation faulting or slip by partials and is based on the idea suggested by Hu et al. [7] to explain the transition from Copper to Brass-type textures in low SFE fcc materials. The original mechanism involves glide on a fixed $\{111\}$ slip plane in either the conventional $\langle 110\rangle$ or partial $\langle 112\rangle$ directions. In other words, the $\{111\}\langle 112\rangle$ systems do not contribute to deformation accommodation as twinning systems, but as partial slip systems with reversible glide along the $\langle 112\rangle$ direction. Perfect slip was also suppressed in Case II, such that only $24\{111\}\langle 112\rangle$ partial slip systems (counting both forward and reverse slip directions) were considered, in order to isolate the effect of deformation faulting from perfect slip. A similar approach was adopted previously by Saleh et al. [1] and Beyerlein et al. [8] during the Visco-Plastic SelfConsistent modelling of monotonic tensile loading (TWIP steel) and rolling (Ag-Cu cast eutectic nanocomposite) textures in low SFE fcc materials.

It is emphasized that while the above two modelling cases assess different aspects of TWIP steel deformation behaviour, the physical reality of deformation accommodation is the result of the concurrent and competing interaction between perfect/partial slip and twinning.

\section{Results and Discussion}

The Voce hardening parameters (Table 1) were adjusted until optimal agreement with the macroscopic stress-strain was achieved for Cases I and II (Fig. 1). In Fig. 1a only Case II is shown for brevity. The experimental hysteresis loop is generally well captured such that the simulated macroscopic flow stress tends to saturate with further cycling and it closely follows the gradual elastoplastic transition upon load reversal. As seen in Fig. 2 (and similar to Case I predictions in Fig. 7, Ref. [3]), Case II also returns good agreement with the lattice strains such that the shape, magnitude and width of the lattice strain hysteresis loops of the $\{111\},\{200\}$ and $\{220\}$ grain families are reasonably predicted.

The adequacy of Cases I and II in describing the underlying deformation mechanisms is assessed via two experimental features: (i) the Bauschinger effect or early yielding during unloading (Fig. 1b) and (ii) the tension-compression asymmetry (Fig. 1c). 
With respect to the early yielding and the associated elasto-plastic transition upon load reversal (Fig. 1b), it is seen that Case I provides reasonable agreement with the experimental macroscopic stress-strain curve as it accounts for the stress relaxation effect incorporated in the twinning scheme; such that back stress is enforced between the parent and the twin upon the creation of the latter. However the partial slip approach of Case II follows the macroscopic stress-strain curve even more closely than Case I during unloading as it mimics the early reverse yielding of the experimental data.

Prior to twin formation, i.e., before the divergence of the dissociated partial dislocations to an infinite separation distance with increasing stress [9], the improved agreement of Case II is in accordance with the stress-induced separation of partial dislocations idea; as slip on the $\{111\}\langle 112\rangle$ systems is allowed to change direction during load reversal. It is argued that while partial dislocations are pulled apart during forward loading, they tend to restore their equilibrium separation distance upon unloading [10]. In turn, both the associated stored energy release and the change in slip direction during load reversal can provide additional sources of back stress in low SFE materials.

Following the initiation of twinning, the improved prediction of Case II raises the possibility that de-twinning events associated with the reversibility of slip along the $\langle 112\rangle$ direction could also be occurring. To the best of our knowledge, no unambiguous observation of de-twinning has been made during reverse loading of low SFE fcc polycrystals. On the other hand, de-twinning of tensile twins has been reported in the hexagonal close-packed (hcp) materials $\mathrm{Mg}$ [11] and $\mathrm{Zr}$ [12] such that twins alternately disappear (or become narrower) and reappear during the subsequent unloading and reloading, respectively. In hcp materials such an effect is made possible by the nearly $90^{\circ}$ twinassociated reorientation of the c-axis during tension which leaves the twinned domain properly oriented for tension and de-twinning upon load reversal. On the other hand, in cubic materials the reoriented domain is not as well oriented for twinning when the stress is reversed. De-twinning was also observed during the cyclic loading of body-centred cubic Ti-V alloys and was associated with an anomalous Bauschinger effect [13].

In one of the only studies pointing out de-twinning in low SFE fcc materials, Doquet [14] detected fragmented twins after reverse torsion of Co-33Ni alloy. Following [13], Doquet argued that the observed twin fragmentation upon load reversal was due to the interaction between dislocations and twins along the twin-matrix interface; which does not allow for the homogenous de-twinning of the whole twin.

Lastly, the above improved prediction of the Bauschinger effect via Case II is provided with the following caveat. As seen in Fig. 1c and detailed in [3], tension-compression asymmetry is expected in materials that deform by twinning due to their inherently lower yield stress in uniaxial tension compared to compression [15]. Here Case I captures the asymmetric behaviour between tension and 
compression as it accounts for both, the irreversibility of twinning and Schmid factor considerations wherein not all the twinning systems activated during tension are activated during compression [15].

On the other hand, while the reversibility of slip along the $\langle 112\rangle$ direction allows Case II to return improved predictions of the Bauschinger effect, the same reversibility (or the absence of directionality of twinning) is the reason behind Case II failing to reproduce the observed tension-compression asymmetry (Fig. 1c). Since twinning is mediated by Shockley partials [9], this raises another intriguing question as to the physical mechanism that justifies Shockley partials being activated in one direction but not in the opposite direction. An answer may lie in the fact that the activation of perfect or partial slip generally follows a resolved shear stress (Schmid factor) law. Here the Schmid factor for twinning is taken as the Schmid factor value of the leading partial dislocation. Since this value is different under tension and compression [16], twinning in fcc materials occurs by shear in one direction but not in the opposite direction. Interestingly, Kibey et al. [17] provided a physical mechanistic basis for the twinning directionality following their $a b$ initio density functional theory calculations. They demonstrated that for the same final twin configuration, one shear direction is favoured over its opposite direction due to the larger energy barriers associated with the unfavourable stacking of atoms in the opposite direction.

\section{Conclusions}

The present study provides an improved prediction of the Bauschinger effect when the reversibility of partial slip in the $\langle 112\rangle$ direction is accounted for (Case II), as it: (i) indicates an important contribution of the stress-induced separation of partial dislocations to the Bauschinger effect, and (ii) for the first time raises the possibility that de-twinning could be operative during the reverse loading of TWIP steel. Further microscopy work is currently underway to verify the above mechanisms.

\section{Acknowledgments}

This work was funded by the Australian Research Council - Discovery Project (DP130101882). Prof. D.B. Santos (UFMG, Brazil) is thanked for the as-cast steel. The access to major research facilities program is supported by the Commonwealth of Australia under the International Science Linkages program. CNT was fully supported by the U.S. DOE project FWP 06SCPE401 under U.S. DOE contract W-7405-ENG-36. This work has benefited from the use of LANSCE which is funded by the U.S. DOE. LANL is operated by Los Alamos National Security LLC under U.S. DOE contract DE-AC52-06NA25396. 


\section{References}

[1] A.A. Saleh, E.V. Pereloma, A.A. Gazder, Microstructure and texture evolution in a twinning-inducedplasticity steel during uniaxial tension, Acta Mater. 61(7) (2013) 2671-2691.

[2] A.A. Saleh, C. Haase, E.V. Pereloma, D.A. Molodov, A.A. Gazder, On the evolution and modelling of brass-type texture in cold-rolled twinning-induced plasticity steel, Acta Mater. 70 (2014) 259-271.

[3] A.A. Saleh, E.V. Pereloma, B. Clausen, D.W. Brown, C.N. Tomé, A.A. Gazder, On the evolution and modelling of lattice strains during the cyclic loading of TWIP steel, Acta Mater. 61(14) (2013) 52475262.

[4] B. Clausen, C.N. Tomé, D.W. Brown, S.R. Agnew, Reorientation and stress relaxation due to twinning: Modeling and experimental characterization for Mg, Acta Mater. 56(11) (2008) 2456-2468.

[5] P.A. Turner, C.N. Tomé, A study of residual stresses in Zircaloy-2 with rod texture, Acta Metall. Mat. 42(12) (1994) 4143-4153.

[6] J.A. Wollmershauser, B. Clausen, S.R. Agnew, A slip system-based kinematic hardening model application to in situ neutron diffraction of cyclic deformation of austenitic stainless steel, Int. J. Fatigue 36(1) (2012) 181-193.

[7] H. Hu, R.S. Cline, S.R. Goodman, Deformation textures of metals, in: H. Margolin (Ed.) American Society of Metals Seminar on Recrystallization, Grain Growth and Textures, Metals Park, Ohio, 1966, pp. 295-367.

[8] I.J. Beyerlein, N.A. Mara, D. Bhattacharyya, D.J. Alexander, C.T. Necker, Texture evolution via combined slip and deformation twinning in rolled silver-copper cast eutectic nanocomposite, Int. J. Plast. 27(1) (2011) 121-146.

[9] T.S. Byun, On the stress dependence of partial dislocation separation and deformation microstructure in austenitic stainless steels, Acta Mater. 51(11) (2003) 3063-3071.

[10] A. Abel, H. Muir, Bauschinger effect and stacking fault energy, Phil. Mag. 27 (1973) 585-594.

[11] L. Wu, S.R. Agnew, D.W. Brown, G.M. Stoica, B. Clausen, A. Jain, D.E. Fielden, P.K. Liaw, Internal stress relaxation and load redistribution during the twinning-detwinning-dominated cyclic deformation of a wrought magnesium alloy, ZK60A, Acta Mater. 56 (2008) 3699-3707.

[12] G. Proust, G. Kaschner, I. Beyerlein, B. Clausen, D. Brown, R. McCabe, C. Tomé, Detwinning of HighPurity Zirconium: In-Situ Neutron Diffraction Experiments, Exp. Mech. 50(1) (2010) 125-133.

[13] S.B. Chakrabortty, T.K. Mukhopadhyay, E.A. Starke Jr, The cyclic stress-strain response of titanium-vanadium alloys, Acta Metall. 26(6) (1978) 909-920.

[14] V. Doquet, Twinning and multiaxial cyclic plasticity of a low stacking-fault-energy f.c.c. alloy, Acta Metall. Mat. 41(8) (1993) 2451-2459.

[15] W.F. Hosford, T.J. Allen, Twinning and directional slip as a cause for a strength differential effect, Met. Trans. 4 (1973) 1424-1425.

[16] C. Efstathiou, H. Sehitoglu, Strain hardening and heterogeneous deformation during twinning in Hadfield steel, Acta Mater. 58(5) (2010) 1479-1488.

[17] S. Kibey, J.B. Liu, D.D. Johnson, H. Sehitoglu, Energy pathways and directionality in deformation twinning, Appl. Phys. Lett. 91(18) (2007) 181916-3. 


\section{Figures}
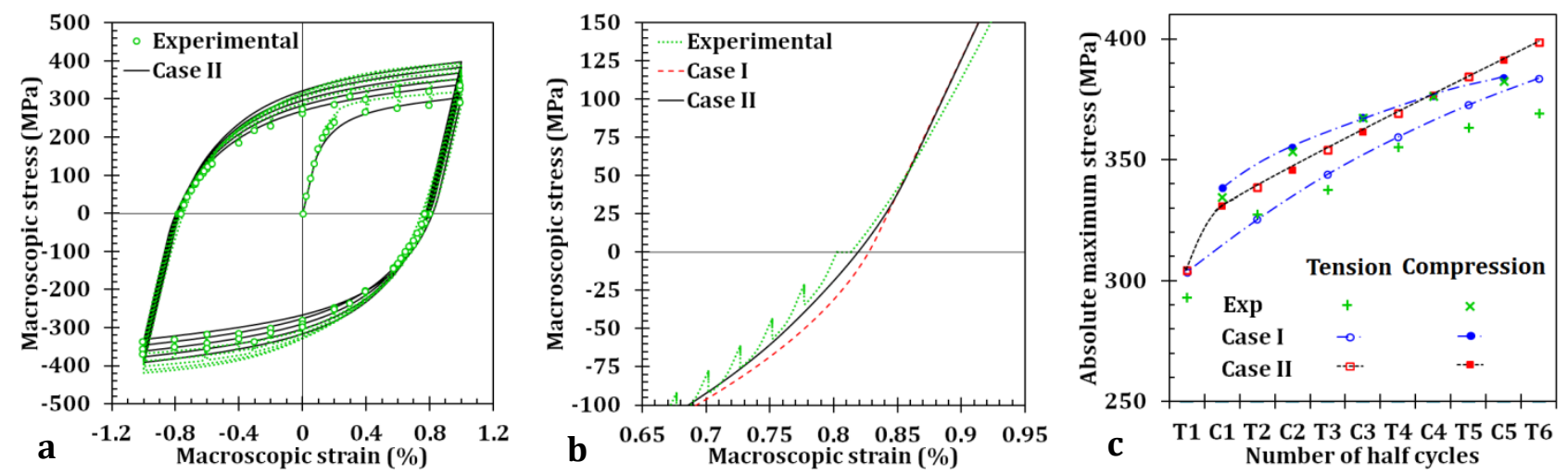

Figure 1. (a) The experimental and EPSC simulated macroscopic stress-strain curves for Case II. (b) A zoomed-in view of the stress-strain curve when reversing from tension to compression during the first cycle. (c) Comparison between Cases I and II predictions of the evolution of the maximum tensile and compressive stresses.
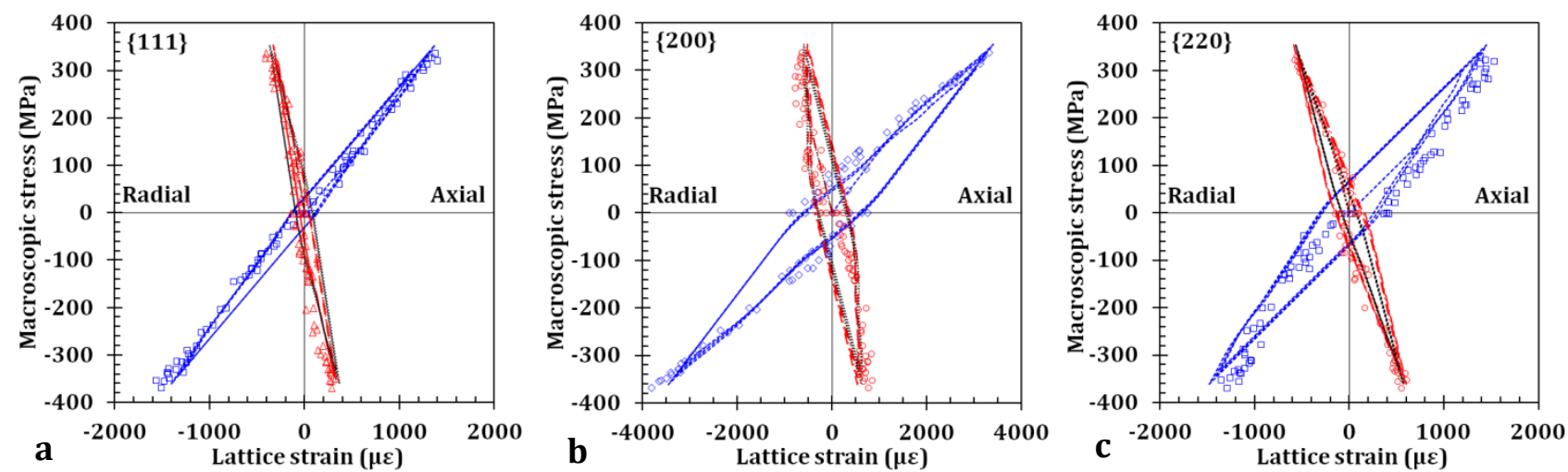

Figure 2. The experimental and EPSC simulated (Case II) evolution of the lattice strain in the axial and radial directions during the first three cycles for the (a) $\{111\}$, (b) $\{200\}$ and (c) $\{220\}$ grain families. The red dashed line $=$ transverse direction and the dotted black line $=$ normal direction . 


\section{$\underline{\text { Tables }}$}

Table 1. The Voce hardening parameters used in the EPSC model for slip and twinning/partial slip systems according to Eqs. (1 and 2). All units are in MPa.

\begin{tabular}{clcccccccc}
\hline \multirow{2}{*}{ Case } & Hardening rule & \multicolumn{3}{c}{ Slip $\{111\}\langle 110\rangle$} & \multicolumn{3}{c}{ Twin/Partial slip $\{111\}\langle 112\rangle$} \\
\cline { 2 - 10 } & & $\tau_{0}^{s}$ & $\tau_{1}^{s}$ & $\theta_{0}^{s}$ & $\theta_{1}^{s}$ & $\tau_{0}^{s}$ & $\tau_{1}^{s}$ & $\theta_{0}^{s}$ & $\theta_{1}^{s}$ \\
\hline \multirow{2}{*}{$I^{*}$} & Forest (Isotropic) & 63 & 20 & 100 & 20 & 111 & 60 & 100 & 20 \\
\cline { 2 - 10 } & Back stress (Kinematic) & - & 54 & 27000 & 100 & - & - & - & - \\
\hline \multirow{2}{*}{ II } & Forest (Isotropic) & - & - & - & - & 62 & 30 & 65 & 40 \\
\cline { 2 - 10 } & Back stress (Kinematic) & - & - & - & - & - & 38 & 35000 & 1100 \\
\hline
\end{tabular}

* In Case I the kinematic hardening parameters for twinning were set to zero as Eq. (2) is not applicable to unidirectional twinning. 